\title{
Efeito do ácido indolbutírico sobre estacas apicais e medianas de quaresmeira (Tibouchina fothergillae Cogn.)
}

\author{
MÁRCIA DE NAZARÉ OLIVEIRA RIBEIRO (1); PATRÍCIA DUARTE DE OLIVEIRA PAIVA (2); \\ JOSÉ DA CONCEIÇÃO BARBOSA SILVA ${ }^{(3)}$ e RENATO PAIVA ${ }^{(4)}$
}

\begin{abstract}
RESUMO
As quaresmeiras, como são conhecidas algumas espécies do gênero Tibouchina, são plantas nativas do Brasil, de porte arbóreo ou arbustivo, muito utilizadas em projetos paisagísticos. A propagação pode ser feita através sementes e estacas. Objetivou-se neste trabalho verificar a influência das concentrações de ácido indolbutírico e diferentes tempos de imersão sobre estacas apicais e medianas de quaresmeira-arbustiva (Tibouchina fothergillae). Estacas medianas e apicais foram coletadas de ramos maduros e cortadas em segmentos com três nós e duas folhas, com metade da área foliar. Estas foram tratadas com AIB, na forma líquida, nas concentrações de 0, 500, 1000 e 2000 mg. $\mathrm{L}^{-1}$, durante 0, 1 e 5 minutos de imersão. Logo após, foram plantadas em bandejas, preenchidas com areia em casa-de-vegetação com nebulização intermitente, onde permaneceram por 56 dias. Pelos resultados obtidos, concluiu-se que o AIB aumentou o número de folhas formadas em estacas apicais até a concentração de 812,5 mg. $\mathrm{L}^{-1}$, também houve influência da concentração de AIB sobre a altura das plantas, número e comprimento dos brotos, tanto em estacas apicais como medianas. Maior comprimento de raiz em estacas medianas foi observado na concentração de $1225 \mathrm{mg} . \mathrm{L}^{-1}$ de AIB. Não há necessidade do uso do ácido indolbutírico no processo de enraizamento para esta espécie.
\end{abstract}

Palavras-chave: Estaquia, AIB, Melastomataceae.

\begin{abstract}
Effect of indolbutiric acid on medium and apical cuttings of lenten tree (Tibouchina fothergillae Cogn.)

Lenten trees, as known some of the species of the genus Tibouchina, are native plants from Brazil used in landscape design projects. The species can be propagated through seeds and cuttings. The objective of this work was to study the influence of indolbutiric acid concentrations and different immersion times on apical and medium cuttings of Tibouchina fothergillae. Medium and apical cuttings were collected from mature branches and cut in segments with three nodes and two leaves with half of the leaf area. Cuttings were treated with IBA, in concentrations of 0, 500, 1000 and $2000 \mathrm{mg} . \mathrm{L}^{-1}$, for 0 , 1 and 5 minutes of immersion. The cuttings were placed in a plastic tray having sand as a substrate and moved to a green house with intermittent vapor irrigation during 56 days. The results showed that IBA increased the number of leaf formed in apical cuttings up to the concentration of $812,5 \mathrm{mg} . \mathrm{L}^{-1}$. There was influence of IBA on height of plants, number and length of sprout, in medium and apical cuttings. Larger root of length in medium cuttings was observed to the concentration of $1225 \mathrm{mg} . \mathrm{L}^{-1}$ of IBA. Thus, the IBA did not influence the rooting process in this species.
\end{abstract}

Key-words: Cuttings, IBA, Melastomataceae.

\section{INTRODUÇÃO}

O gênero Tibouchina, pertence à família Melastomataceae e sua floração coincide com a Quaresma, daí vem o nome popular das espécies conhecidas como quaresmeiras (SMITH, 2000). Esta família não tem grande valor econômico, seus usos incluem ornamentais (Tibouchina), frutos comestíveis (Bellucia), madeira para construção (Astronia), tintas (Bellucia) (RIBEIRO et al., 1999), servindo ainda, como indicadoras de poluição (KLUMPP et al., 2000a,b; MORAES et al., 2000a,b).

As espécies mais conhecidas desta família são árvores de até $12 \mathrm{~m}$ e arbustos de cerca de $2 \mathrm{~m}$ nativos do Brasil e freqüente nos estados do Rio de Janeiro, São Paulo, Minas Gerais, Bahia, Santa Catarina e Pará (GRANDE
ENCICLOPÉDIA BARSA, 2004). A Tibouchina fothergillae é uma espécie arbustiva e se diferencia de outras espécies de Melastomataceae por suas flores terem coloração "vinho" intenso (figura 1), as quais nas outras espécies normalmente são arroxeadas, sendo assim, apresenta características ornamentais interessantes para uso em projetos paisagísticos.

Segundo LORENZI (2003), a propagação das quaresmeiras pode ser feita através de sementes ou estacas, porém, as sementes são muito pequenas, semelhantes à areia fina, não tolerantes ao armazenamento e com baixa taxa de germinação.

A propagação vegetativa é amplamente utilizada na produção de mudas de plantas ornamentais (BARBOSA, 2003), principalmente para multiplicar

\footnotetext{
(1) M.Sc., Doutoranda em Agronomia/Fitotecnia, Universidade Federal de Lavras (UFLA/DAG), Lavras-MG, E-mail:mribeiro@ufla.br

(2) D.Sc., Prof. Adjunto, Universidade Federal de Lavras (UFLA/DAG), Cx. P. 3037, 37200-000, Lavras-MG.

(3) Graduado em Ciências Agrárias, Centro Federal Tecnológico (CEFET-MA), São Luís-MA.

(4) Ph.D, Prof. Adjunto,Universidade Federal de Lavras (UFLA/DBI), Lavras-MG.
} 
plantas que não produzem sementes botânicas ou sementes com baixa viabilidade, em pequeno número ou, ainda, para acelerar a produção de mudas de espécies que apresentam problemas de germinação, como aquelas que produzem sementes dormentes (PASQUAL, 2002). A propagação vegetativa pode ser realizada via estaquia, enxertia, mergulhia, estolões, bulbos, divisão de touceiras e por cultura de tecidos.

A estaquia destaca-se por promover a multiplicação de plantas matrizes, agronomicamente superiores, mantendo-se as suas características desejáveis (MELETTI, 2000), bem como para a obtenção de uma população clonal geneticamente uniforme, em curto espaço de tempo, com redução ou eliminação da fase juvenil das plantas cultivadas (JANICK, 1966).

A viabilidade do uso da estaquia na propagação comercial é em função da facilidade de enraizamento de cada espécie e/ou cultivar, da qualidade do sistema radicular formado e do desenvolvimento posterior da planta na área de produção (DUTRA \& VILLA, 2004). Contudo, combinando-se uma ou mais técnicas auxiliares, como a nebulização intermitente e a aplicação de fitorreguladores, entre outros, os resultados poderão ser mais satisfatórios (FACHINELLO et al., 2005).

A principal classe de fitorreguladores usados no enraizamento de estacas são as auxinas, sendo o AIB o mais indicado, pois não apresenta toxidade em uma larga faixa de concentração, além de apresentar baixa mobilidade e maior estabilidade química no corpo das estacas (PASQUAL, 2002).

A escolha do tipo de estaca a ser utilizada tem grande importância, principalmente, para aquelas espécies com dificuldades de formar raízes adventícias. Em relação à posição ocupada no ramo de origem, as estacas podem ser apicais (ou terminais), medianas ou basais. Existem diferenças marcantes na composição química da base ao ápice dos ramos e, assim, são observadas variações na formação de raízes de estacas obtidas de diferentes partes dos ramos (OLIVEIRA et al., 2001).

Em geral, estacas apicais apresentam nível de auxina mais alto que as estacas intermediárias ou medianas. Mesmo assim, a aplicação externa de reguladores de crescimento, como o AIB, pode melhorar e uniformizar o enraizamento. Estacas medianas necessitam maior período para regenerar nova planta, o que se deve em geral ao fato das gemas laterais estarem geralmente dormentes. Em muitos casos, antes de ocorrer o enraizamento, há o desenvolvimento das ramificações laterais, com formação de ramos jovens, às custas de reservas nutricionais armazenadas no corpo da estaca (KAMPF, 2000).

A presença de folhas em estacas exerce forte influência estimuladora na formação de raízes. Os carboidratos, resultantes de atividades fotossintéticas das folhas, também contribuem para a formação de raízes, embora os efeitos estimuladores de folhas e gemas se devam, principalmente, à produção de auxina. O tratamento de estacas com auxinas têm como objetivos: aumentar a porcentagem de estacas que formam raízes, ou acelerar sua formação ou, ainda, aumentar o número e a qualidade das raízes formadas em cada estaca (GOULARD, 2003).

O objetivo deste trabalho foi verificar o efeito de diferentes concentrações de ácido indolbutírico e tempos de imersão no processo de enraizamento e brotação em estacas apicais e medianas, e na formação de mudas de Tibouchina fothergillae.

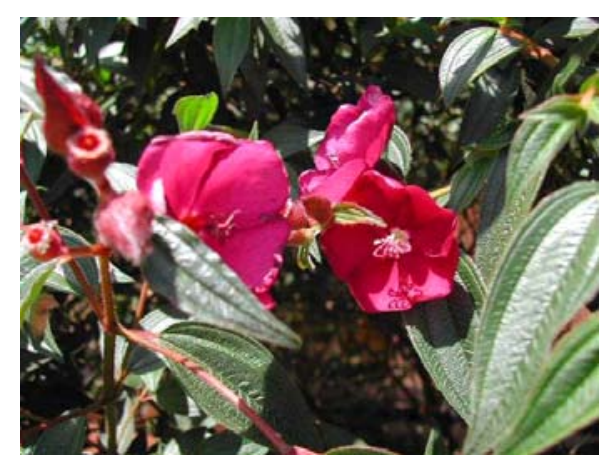

Figura 1. Flores de Tibouchina fothergillae Cogn.

Figure 1. Flowers of Tibouchina fothergillae Cogn.

\section{MATERIALEMÉTODOS}

O trabalho foi realizado em casa-de-vegetação equipada com sistema de nebulização intermitente, no Departamento de Agricultura da Universidade Federal de Lavras (UFLA). Foram utilizadas estacas de ramos de Tibouchina fothergillae, retiradas de matrizes adultas do Viveiro de Plantas Ornamentais da referida Universidade.

As estacas medianas e apicais, obtidas após a coleta dos ramos, constituíram dois experimentos separados. Nas estacas medianas (terço médio do ramo) realizou-se um corte transversal abaixo da gema, na base, e em bisel na parte superior; e nas estacas apicais (parte apical do ramo) foi realizado apenas um corte transversal abaixo da gema localizada na base. As estacas foram padronizadas com três nós e um par de folhas, sendo estas cortadas pela metade. Posteriormente, as estacas foram tratadas com solução de ácido indolbutírico, nas concentrações de 0, 500, 1000 e 2000 mg.L - $^{-1}$ em diferentes tempos: 0, 1 e 5 minutos. O tratamento testemunha constituiu-se de imersão da base das estacas em água destilada.

As estacas, após a imersão, foram colocadas em bandejas de polipropileno de 72 células contendo areia 
como substrato. Os experimentos foram conduzidos em delineamento inteiramente casualizado com 8 repetições e 10 estacas por parcela, constituindo fatorial simples 4 (concentrações de AIB) x 3 (tempos de imersão). O material foi mantido em estufa com nebulização intermitente. Os resultados foram submetidos à análise de variância, utilizando o software Sisvar (FERREIRA, 2000).

O período experimental teve duração de 56 dias, ao final do qual avaliou-se: porcentagem de enraizamento, altura da planta, número de folhas formadas, número de brotos e comprimento dos brotos para estacas apicais. Para estacas medianas avaliou-se: porcentagem de enraizamento, altura da planta, número de brotos, comprimento dos brotos e o comprimento da maior raiz.

\section{RESULTADOS E DISCUSSÃO}

Para Tibouchina fothergillae, o uso do regulador de crescimento AIB não influenciou o

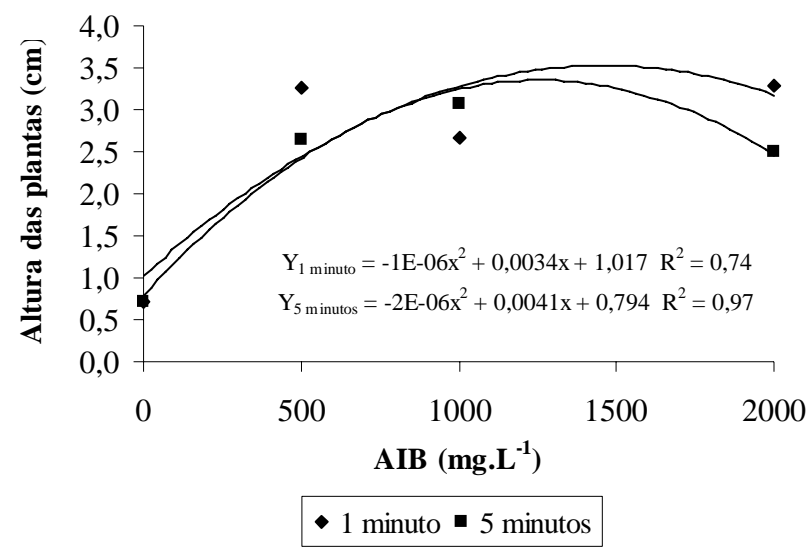

Figura 2A. Altura das plantas de Tibouchina fothergilla, em diferentes concentrações de ácido indolbutírico (AIB), em 1 e 5 minutos de imersão das estacas apicais.

Figure 2A. Height of Tibouchina fothergilla plants in different concentrations of indolbutiric acid (IBA), after immersion of apical cuttings for 1 and 5 minutes. enraizamento, pois as estacas usadas como testemunha também tiveram boa formação de raízes. Para esta espécie houve $94 \%$ de enraizamento, tanto para estacas apicais, como para estacas medianas.

Maior altura das plantas em estacas apicais $(3,9 \mathrm{~cm})$ foi obtida com a concentração de $1700 \mathrm{mg} . \mathrm{L}^{-1}$ de AIB, em 1 minuto de imersão das estacas. Maior altura das plantas em estacas medianas $(8,8 \mathrm{~cm})$ foi obtido com 1437,5 mg.L ${ }^{-1}$ de AIB em 1 minuto de imersão das estacas (figuras 2A e 2B).

Maior número de folhas formadas em estacas apicais $(2,8)$ foi obtido na concentração de 812,5 mg.L${ }^{1}$ de AIB em imersão rápida das estacas (figura 3).

Maior número de brotos em estacas apicais $(1,5)$ foi obtido na concentração de $1000 \mathrm{mg} . \mathrm{L}^{-1} \mathrm{AIB}$ em imersão rápida das estacas. Maior número de brotos em estacas medianas $(1,8)$ foi obtido na concentração de 1214,3 mg.L - $^{-1}$ de AIB em 1 minuto de imersão (Figuras $4 \mathrm{~A}$ e $4 \mathrm{~B})$.

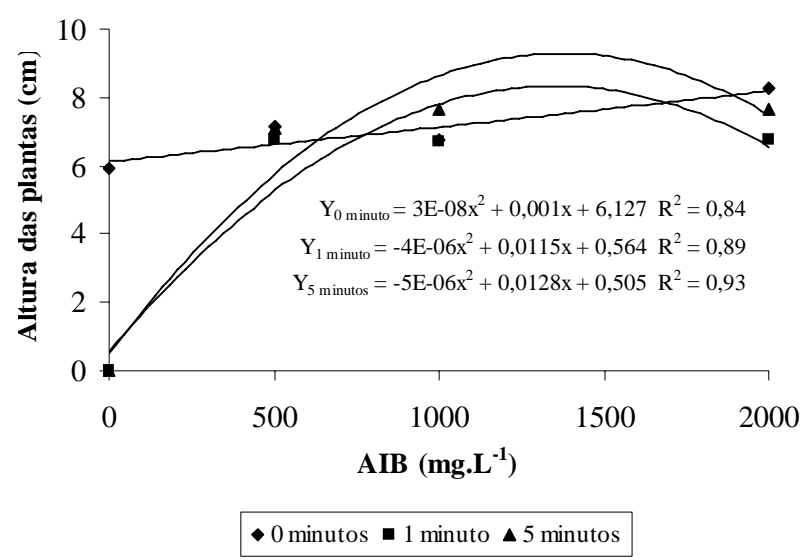

Figura 2B. Altura das plantas de Tibouchina fothergillae, em diferentes concentrações de ácido indolbutírico (AIB), em 0, 1 e 5 minutos de imersão das estacas medianas.

Figure 2B. Height of Tibouchina fothergillae plants in differents concentrations of indolbutírico acid (IBA), after immersion of median cuttings for 0, 1 and 5 minutes.

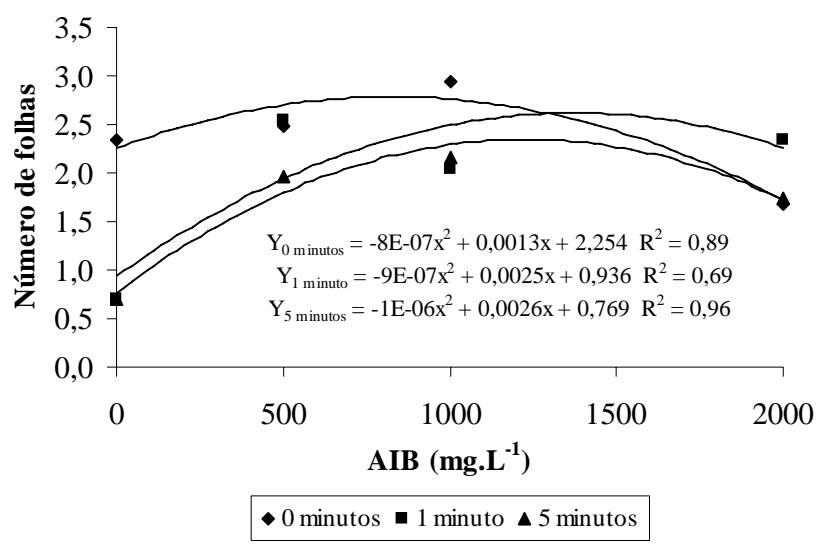

Figura 3. Número de folhas de Tibouchina fothergillae, em diferentes concentrações de ácido indolbutírico (AIB), após imersão das estacas apicais por 0, 1 e 5 minutos.

Figure 3. Leaves number of Tibouchina fothergillae in differents concentrations of indolbutiric acid (IBA), after immersion of apical cuttings per 0, 1 and 5 minutes. 


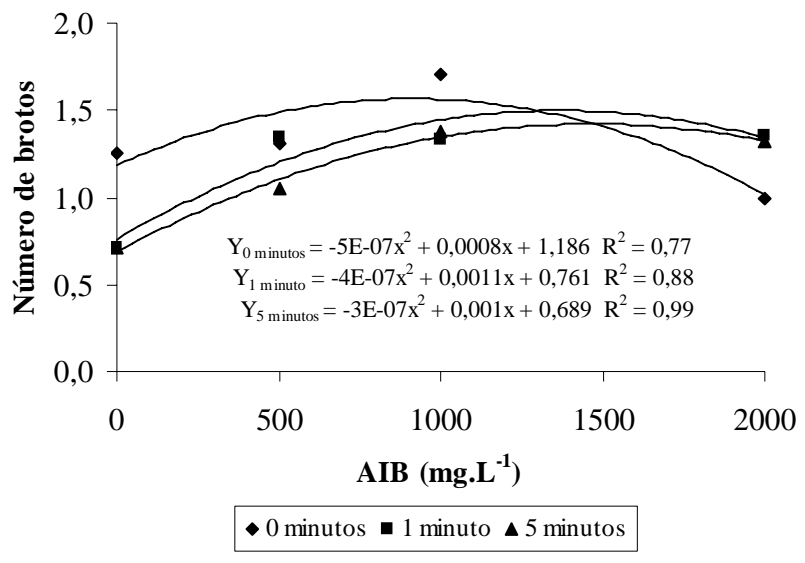

Figura 4A. Número de brotos em estacas apicais de Tibouchina fothergillae em diferentes concentrações de ácido indolbutírico (AIB), em 0, 1 e 5 minutos de imersão. Figure 4A. Sprouts number of Tibouchina fothergillae in differents concentrations of indolbutírico acid (IBA), after 0,1 e 5 minutes of immersion of the apical cuttings.

Maior comprimento dos brotos em estacas apicais $(4,0 \mathrm{~cm})$ foi observado na concentração de $1750 \mathrm{mg} . \mathrm{L}^{-1} \mathrm{de}$ AIB em 1 minuto de imersão das estacas. Maior comprimento dos brotos em estacas medianas $(1,6 \mathrm{~cm})$ foi obtido com 1071,4 mg. - $^{-1}$ de AIB em 5 minutos de imersão das estacas (figuras $5 \mathrm{~A}$ e $5 \mathrm{~B}$ ).

A emissão e alongamento de brotações não devem ser relacionados necessariamente com o enraizamento, pois muitas vezes, os brotos, ao invés de contribuírem, prejudicam a iniciação radicular à medida que passam a competir pelas reservas das estacas, resultando na formação dos brotos em detrimento das raízes, provocando a desidratação do material propagativo através da transpiração (HOWARD et al., 1984).

Maior comprimento de raiz em estacas medianas (3,9 cm) foi observado com $1225 \mathrm{mg} \cdot \mathrm{L}^{-1}$ de AIB, em 5 minutos de imersão das estacas (figura 6). Com a aplicação

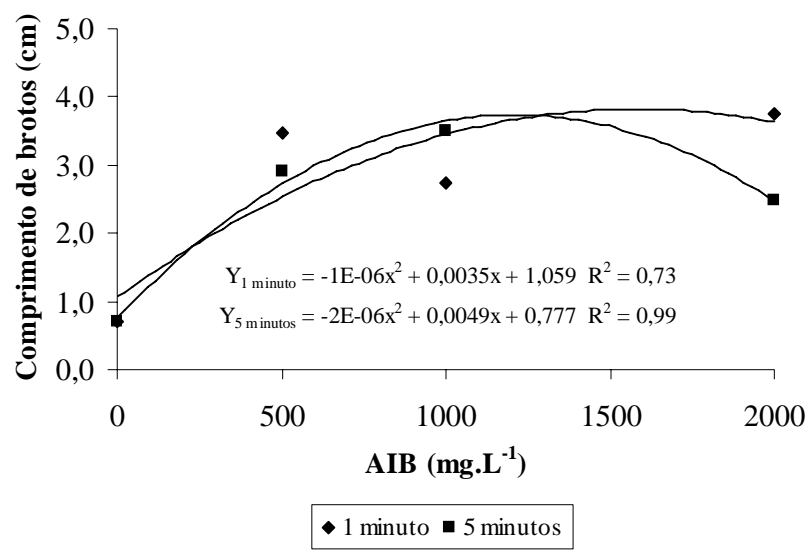

Figura 5A. Comprimento dos brotos de Tibouchina fothergillae, em diferentes concentrações de ácido indolbutírico (AIB), em 1 e 5 minutos de imersão de estacas apicais

Figure 5A. Sprout length of Tibouchina fothergillae, in differents concentrations of indolbutiric acid (IBA) in 1 and 5 minutes of immersion apical cuttings.

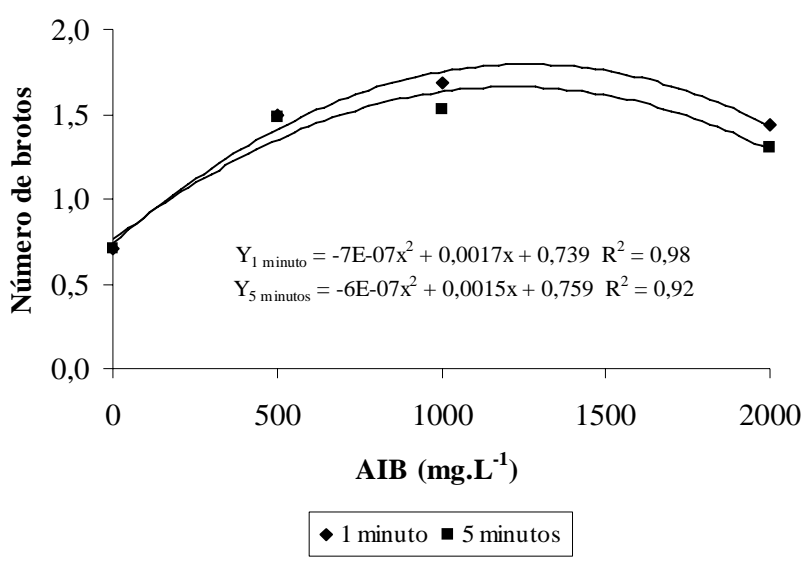

Figura 4B. Número de bortos de Tibouchina fothergillae, em diferentes concentrações de ácido indolbutírico (AIB), após 1 e 5 minutos de imersão das estacas medianas.

Figure 4B. Sprouts number of Tibouchina fothergillae, in differents concentrations of indolbutírico acid (IBA), after 1 and 5 minutes of immersion of median cuttings.

de auxina, há um aumento da concentração na base da estaca, estimulando a formação do calo, que é o resultado da ativação das células do câmbio e das raízes adventícias (FACHINELLO et al., 1995).

TOFANELLI (1999) observou incremento no comprimento de raízes à medida que se aumentou a concentração de AIB, aplicada em estacas lenhosas de pessegueiro 'Arlequin', 'Biuti', 'Maravilha', 'Premier', 'Okinawa' ' 'R-15-2'. BARTOLINI \& ROSELLI (1975) e SHARMA \& AIER (1989), também observaram influência do AIB no comprimento da maior raiz de ameixeira.

O teor adequado de auxina exógena, para estímulo de enraizamento, depende da espécie e da concentração da auxina existente no tecido. É necessário que haja um balanço adequado entre auxinas, giberelinas e citocininas, ou seja, equilíbrio entre promotores e inibidores do processo de brotação e iniciação radicular (FACHINELLO et al. 2005).

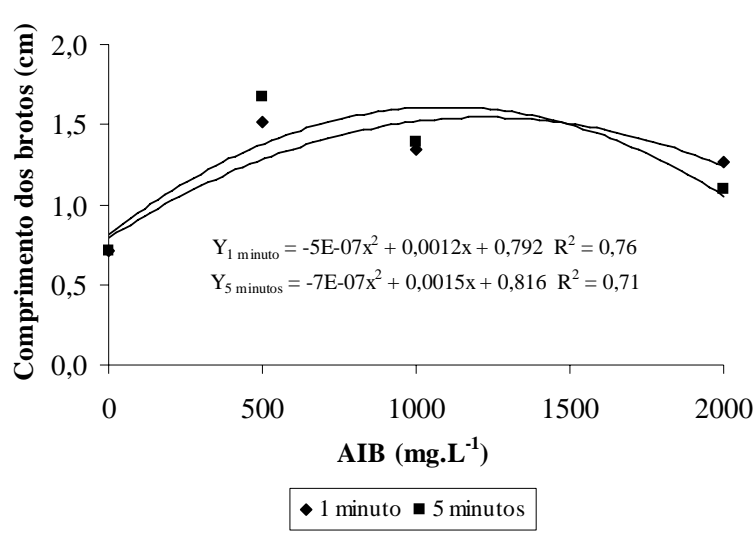

Figura 5B. Comprimento dos brotos de Tibouchina fothergillae, em diferentes concentrações de ácido indolbutírico (AIB), em 1 e 5 minutos de imersão de estacas medianas.

Figure 5B. Sprout length of Tibouchina fothergillae in differents concentrations of indolbutiric acid (IBA), in 1 and 5 minutes of immersion of mediun cuttings. 


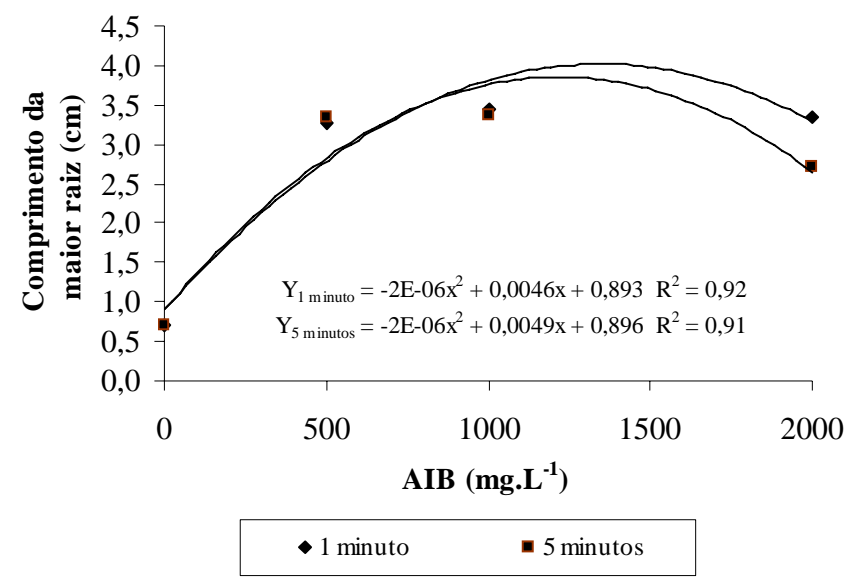

Figura 6. Efeito das concentrações de AIB para comprimento da maior raiz em estacas medianas de Tibouchina fothergillae, em 1 e 5 minutos de imersão.

Figure 6. Effetc of indolbutiric acid (IBA) concentrations for length high root in medium cuttings of Tibouchina fothergillae, in 1 and 5 minutes of immersion.

As mudas, depois de enraizadas, foram transferidas para vasos com substrato. Observou-se nessa fase bom pegamento das mudas formadas de estacas apicais e, ao contrário, houve morte de $100 \%$ das mudas oriundas de estacas medianas, o que evidencia a necessidade de outros estudos para mais esclarecimentos.

\section{CONCLUSÕES}

1. Para o enraizamento desta espécie recomendase a utilização de estacas apicais;

2. O AIB não interferiu no enraizamento, não sendo necessário o seu uso, pois as estacas sem este fitorregulador (testemunha) enraizaram;

3. Na fase de transferência para vasos, não houve pegamento das mudas oriundas de estacas medianas.

\section{REFERÊNCIAS BIBLIOGRÁFICAS}

BARBOSA, J.G. Crisântemo: produção de mudas - cultivo para corte de flor - cultivo em vaso - cultivo hidropônico. Viçosa: Aprenda Fácil, 2003. 234p.

BARTOLINI, G; ROSELLI, G. Richerche sulla propagazione del susino per tale adi ramo: 2-moltiplicazione di alcune cultivar de Prunus domestica L . fornite di radici avventizie preformate. Revista della Ortoflorofrutticoltura Italiana, Florence, v. 59, n. 5, p. 340-347, 1975.

DUTRA, L.F.; VILLA, F. Estaquia. In: PASQUAL, M. Propagação de Plantas Ornamentais - Curso de pósgraduação “Latu Sensu”. Lavras: UFLA/FAEPE, 2004. 106 p. FACHINELLO, J.C.; HOFFMANN, A.; NACHTIGAL, J.C.; KERSTEN, E.; FORTES, G.R. de L. Propagação de plantas frutíferas de clima temperado. 2. ed. Pelotas: UFPEL, 1995. 178p.

FACHINELLO, J.C.; HOFFMANN, A.; NACHTIGAL, J.C.; KERSTEN, E. Propagação vegetative por estaquia. In: FACHINELLO, J.C.; HOFFMANN, A.; NACHTIGAL, J.C. (Eds). Propagação de plantas frutíferas, Brasília: EMBRAPA, 2005. p. 69-108.

FERREIRA, D.F. Análises estatísticas por meio do Sisvar para Windows versão 4.0. In: REUNIÃO ANUAL DA
REGIÃO BRASILEIRA DA SOCIEDADE INTERNACIONAL DE BIOMETRIA, 45., São Carlos, 2000. Anais..., São Carlos: UFSCar. 2000. p. 255-258.

GOULART, P.B. Desenvolvimento de metodologia para enraizamento de estacas de candeia (Eremanthus erythropappus) (DC) MacLeisch. 2003. 32f. Monografia (Departamento de Engenharia Florestal) - Universidade Federal de Lavras, Lavras, MG.

GRANDE ENCICLOPÉDIA BARSA. $3^{\text {a }}$ ed. São Paulo: Barsa Planeta Internacional Ltda, v. 12, 2004. p. 135.

HOWARD, B.H.; HARRISON-MURRAY, R.S.; MALKEZIE, K.A.D. Rooting responses to wounding winter cutting of M-26 apple rootstock. Journal of Horticultura Science, London, v. 59, n. 2, p. 131-9. 1984. JANICK, J. A ciência na horticultura. Rio de Janeiro: F. Bastos, 1966. 485p.

KAMPF, A.N. Produção Comercial de Plantas Ornamentais. Guaíba: Agropecuária, 2000. 254p.

KLUMPP, A.; DOMINGOS, M \& PIGNATA, M.L. Air pollution and vegetation damage in South America - State of knowledge and perspectives. In: AGRAWAL, S.B.; AGRAWAL, M. (Eds). Environmental pollution and plant responses, Lewis Publishers: CRC Press, New York, 2000a. p. 111-136.

KLUMPP, G.; FURLAN, C.M.; DOMINGOS, M \& KLUMPP. A. Response of stress indicators and growth parameters of Tibouchina pulchra Cogn. exposed to air and soil pollution near the industrial complex of Cubatão, Brazil. The Science of the Total Environment, v. 246, p. 7991. 2000b.

LORENZI, H.; SOUSA, H.M. Plantas Ornamentais no Brasil: arbustivas, herbáceas e trepadeiras. Nova Odessa: Editora Plantarum, 2003. 720p.

MELETTI, L.M.M. Propagação de frutíferas tropicais. Guaíba: Agropecuária, 2000. 239p.

MORAES, R.M.; DELITTI, W.B.C \& MORAES, J.A.P.V. Fotossíntese líquida e crescimento de Tibouchina pulchra Cogn. nas proximidades de indústrias petroquímicas , em Cubatão, SP. Hoehnea, v. 27, p. 77-85. 2000a.

MORAES, R.M.; DELITTI, W.B.C \& MORAES, J.A.P.V. Resposta de indivíduos jovens de Tibouchina pulcha 
Cogn. à poluição aérea de Cubatão, SP: fotossíntese líquida, crescimento e química foliar. Revista Brasileira de Botânica, v. 23, p. 441-447. 2000b.

OLIVEIRA, M.C. de; RIBEIRO, J.F.; RIOS, M.N. da S.; REZENDE, M.E. Enraizamento de Estacas para Produção de Mudas de Espécies Nativas de Matas de Galeria. Brasília: EMBRAPA, 2001. 4p. (Recomendação Técnica, 41).

PASQUAL, M. Propagação de plantas ornamentais. Lavras: UFLA/FAEPE, 2002. 80p.

RIBEIRO, J.E.L da S, et al. Flora da reserva Ducke: guia de identificação das plantas vasculares de uma floresta de terrafirme na Amazônia Central. Manaus: INPA, 1999. 816p.
SHARMA, S.D.; AIER, N.B. Seasonal rooting behaviour of cuttings of plum cultivars as influenced by IBA treatments. Scientia Horticulturae, Amsterdam, v. 40, n. 4, p. 297-303, Nov. 1989.

SMITH, J.I. Árvores ornamentais na cidade de São Paulo. $1^{a}$ ed. São Paulo: Editora Terceiro Nome. 2000. 90p.

TOFANELLI, M.B.D. Enraizamento de estacas lenhosas e semilenhosas de cultivares de pessegueiro em diferentes concentrações de ácido indolbutírico. 1999. 87p. Dissertação (Mestrado em Agronomia) - Universidade Federal de Lavras, Lavras, MG. 